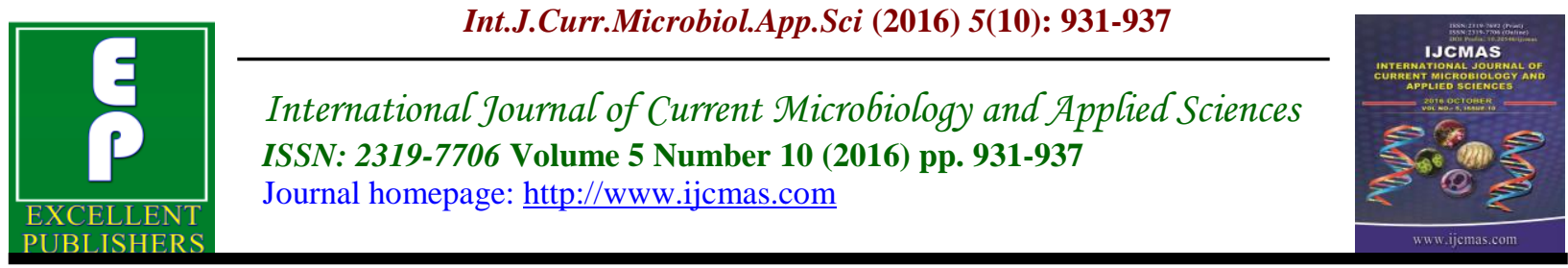

Original Research Article

http://dx.doi.org/10.20546/ijcmas.2016.510.100

\title{
Comparative Analysis of Rapid Dengue Testing and ELISA for NS1 Antigen and IgM in Acute Dengue Infection
}

\author{
R. Mahesh Reddy*, Kavita Sahai, Ajay Malik, S. Shoba and Anurag Khera \\ Department of Microbiology, Armed Forces Medical College \\ Pune - 411048, Maharashtra, India \\ *Corresponding author
}

\begin{abstract}
A B S T R A C T
Keywords

Acute dengue infection, NS1 antigen, $\operatorname{IgM}$ antibody, rapid immunochromatog raphy, MAC ELISA.

Article Info

Accepted:

28 September 2016

Available Online:

10 October 2016

Dengue cases in India are increasing in epidemic proportions. The diagnosis of acute dengue infection is made by viral isolation and identification, viral nucleic acid detection, and serological tests for NS1 Antigen or IgM or IgG seroconversion. Viral isolation, viral nucleic acid detection requires specialized laboratory equipment and expertise. For resource limited labs tests like rapid immunochromatography (ICT) based or ELISA based tests for detection of NS1 antigen or IgM antibody can be used. In this present study we attempted to do a diagnostic test evaluation of rapid ICT with ELISA for detection of NS1 antigen (in $<5$ days fever) and IgM antibody (in $>5$ days fever)for acute dengue diagnosis. The serum samples from the first group $(n=740)$ were subjected to NS1 antigen detection by rapid and ELISA and that of the second group $(n=609)$ were subjected to IgM detection by rapid and IgM capture ELISA. The sensitivity of the rapid ICT tests for NS1 Ag \& IgM in our study was more than $90 \%$, and specificity more than $98 \%$.We noted that rapid ICT kits that we used for testing NS1Ag and IgM performed at par with ELISA based tests.
\end{abstract}

\section{Introduction}

Dengue is one of the vector borne viral disease prevalent worldwide. During the last half a century, the incidence of dengue has increased by 30-fold and expanded to many new countries, and also from urban areas to rural areas (WHO 2009). It is estimated approximately 294 million in apparent dengue infections and about 96 million apparent dengue infections occurred worldwide in 2010, and Asia has contributed to $70 \%$ of this burden. The vector control effects are failing in urban areas which is reflected as rapid yearly rise in the number of dengue cases and spread to suburban and rural areas (Bhatt et al., 2013). Dengue cases in India are rapidly rising every year and in 2015 as per National Vector Borne Disease Control Programme (NVBDCP) a total of 99,913 of dengue have been detected with 220 deaths recorded, and till September 2016 a total of 45490 cases with 88 deaths have been recorded(NVBDCP).

Dengue is caused by any of four serotypes of dengue virus (DENV-1, 2, 3 and 4). Dengue virus is a single- stranded, positive 
sense enveloped RNA virus that belongs to the genus Flavivirus (Pal et al., 2014). Dengue virus infection in humans is often in apparent but can lead to a wide range of clinical manifestations, from mild fever to potentially fatal dengue shock syndrome. Dengue like illness is produced by many viral infections such as Japanese encephalitis (JEV), chikungunya (CHIKV), and West Nile virus (WNV). So, clinically suspected and lab confirmed cases have two different implications (Capeding et al., 2015). Efficient and accurate diagnosis of dengue is of primary importance for clinical care i.e. for early detection of severe cases, case confirmation and to rule out other differential diagnosis. It is also important for the disease surveillance and outbreak control. An approximate time-line of primary and secondary dengue virus infections and the diagnostic methods that can be used to detect infection is depicted in the Fig 1 (WHO, 2009). The World Health Organization (WHO) 2009 guidelines identified three diagnostic tests as golden standards for dengue diagnosis: viral isolation and identification, viral nucleic acid detection, and serological tests for $\operatorname{IgM}$ or $\mathrm{IgG}$ seroconversion.

However, some of these methods have limitations. For example, viral isolation is difficult to perform and time-consuming. Viral nucleic acid detection such as RT-PCR requires specialized laboratory equipment and expertise. Serological tests for $\mathrm{IgG}$ cannot be used for early onset disease diagnosis. Therefore, a more efficient and accurate detection method for Dengue infection is warranted. Non-structural protein 1 (NS1) is a glycoprotein that is abundantly produced by DENV in the early stage of infection, and can be detected in the serum or plasma of the patients (Shan et al., 2015). After the onset of illness, the NS 1 antigen of virus can be detected in serum or plasma 4-5 days. After 05 days i.e. at the end of the initial phase of infection, IgM or $\mathrm{IgG}$ detection is the choice for diagnosis.

As per case definition of dengue according to Indian national guidelines, a patient is labelled as a 'probable case' if he satisfies the clinical criteria during dengue outbreak or positive non ELISA based immunochromatography tests (ICT) such as NS1 antigen(Ag)ICT / IgM ICT("National Guidelines for Clinical Management of Dengue Fever, Dec 2014."). A case is labelled as 'confirmed' when NS1 Ag / Ig M is positive by ELISA or demonstration of fourfold rise of IgG titre or detection of viral nucleic acids PCR or by culture and isolation of Dengue virus. Hence, for the routine diagnosis in peripheral hospitals we can use either rapid ICT or ELISA for the detection of NS1 Ag, IgM antibodies. But the problem with rapid ICTs is their reliability. Presently, there are varied reports on the sensitivity and specificity of the rapid diagnostic tests that are used to detect NS1 $\mathrm{Ag}$ and the IgM antibody. The aim of this study is to assess performance of rapid ICTs in acute dengue diagnosis. We attempted to assess and evaluate rapid diagnostic tests for NS1 Ag and IgM antibody for acute dengue diagnosis in comparison to ELISA based tests in terms of sensitivity and specificity.

\section{Materials and Methods}

The present study is a diagnostic test evaluation of rapid ICT with ELISA for detection of NS1 antigen (in $<5$ days fever) and $\operatorname{IgM}$ antibody (in $>5$ days fever). It was conducted in a tertiary care dengue sentinel surveillance hospital for a period of two years. Patients of all age groups who had clinical symptoms and signs of acute dengue like illness were included in the study. These patients were divided into two groups, the first group who had the illness duration for 
less than five days duration and the second group who had illness for more than five days and upto 15 days from the onset of illness.

Blood samples were collected under aseptic precautions and serum was separated and stored for further analysis. The serum samples from the first group were subjected to NS1 antigen detection by Rapid ICT (SD Bioline, India) and NS1Ag capture ELISA (Panbio, Australia)tests and that of the second group were subjected to $\operatorname{IgM}$ detection by rapid ICT (Alere, India) and IgM capture ELISA (NIV Pune, India) tests. The tests were performed strictly adhering to the kit manufacturer's instructions. A total of 740samples were processed by rapid and ELISA tests for detection of NS1 Ag, and 609 samples were processed by rapid and ELISA tests for detection of IgM antibody. The patients who tested positive for NS1 Ag or IgM antibody by ELISA were taken as confirmed cases and to be suffering from acute dengue infection. The results were analysed by MedCalc statistical software version 16.8 .

\section{Results and Discussion}

The majority of the patients who were tested were in the early phase of the illness. A total of 354 Of 740 were NS1 Ag ELISA positive and 269 of 609 were $\operatorname{IgM}$ antibody positive. The age distribution for all the NS1 or the IgM antibody confirmed cases by ELISA is given in Fig 2. Maximum number of the cases who were either NS1Antigen positive or IgM antibody positive were between the ages of 10-50 years of age. Gender wise distribution is given in Fig 3. It was noted that there was male preponderance in all the confirmed cases.

When the rapid ICT test for NS1 Ag was compared with the NS1 Antigen capture
ELISA, it showed a sensitivity of $90.11 \%$, specificity of $98.45 \%$, Positive predictive value (PPV) of $98.15 \%$ and Negative predictive value of $91.57 \%$.The rapid ICT test for $\operatorname{IgM}$ when compared with the $\operatorname{IgM}$ capture ELISA, it showed a sensitivity of $92.57 \%$, specificity of $98.53 \%$, Positive predictive value of $98.03 \%$ and Negative predictive value of $94.37 \%$ (Table 1).The overall disease prevalence confirmed by ELISA in the study population is $46.1 \%$. The day wise sensitivity and specificity of Dengue NS1 Antigen rapid ICT test vs NS1 Antigen capture ELISA is given in Fig 4.

Acute dengue is a major health problem in India. It has risen to epidemic proportions and is endemic to many areas, both urban and rural. In our study highest prevalence was seen in the age groups between 11-50 years and with male preponderance which is seen in other studies also (Gupta et al., 2006; Chakravarti et al., 2005; Sarkar et al., 2012). The sensitivity of the rapid ICT tests for NS1 Ag\& IgM in our study was more than $90 \%$, and specificity more than $98 \%$ when compared to ELISA. The positive predictive value of rapid ICT for NS1 Ag and $\mathrm{Ig} \mathrm{M}$ is very high i.e. $98 \%$. This indicates that the probability of patient having acute dengue infection if the tests are positive is almost same as the ELISA based tests. This study finding corroborates with other studies, which have shown the PPV of rapid ICTs to be more than $85 \%$ (Pal et al., 2014; Groen et al., 2000; Shih et al., 2016).The rapid ICTs have a major advantage in that, they are easy to perform, they need less expertise and can be done within minutes (Chaterji et al., 2011).

To make ELISA cost effective a large number of samples need to be processed at one go. To perform ELISA test, lab needs to be equipped with instruments like ELISA washer and reader. 
Table.1 Validity and predictive values of rapid ICT vs ELISA for NS1 Ag and IgM antibody detection

\begin{tabular}{|l|c|c|c|c|}
\hline \multirow{2}{*}{\multicolumn{1}{|c|}{ Statistics }} & \multicolumn{2}{c|}{ Group 1 (Fever < 5days) } & \multicolumn{2}{c|}{ Group 2 (Fever > 5days) } \\
\cline { 2 - 5 } & \multicolumn{2}{|c|}{ VS1 Ag: Rapid ICT vs ELISA } & \multicolumn{2}{c|}{ IgM: Rapid ICT vs ELISA } \\
\hline Sensitivity & $90.11 \%$ & $86.52 \%$ to $93.02 \%$ & $92.57 \%$ & $88.75 \%$ to $95.40 \%$ \\
\hline Specificity & $98.45 \%$ & $96.65 \%$ to $99.43 \%$ & $98.53 \%$ & $96.60 \%$ to $99.52 \%$ \\
\hline $\begin{array}{l}\text { Positive Predictive } \\
\text { Value }\end{array}$ & $98.15 \%$ & $96.03 \%$ to $99.32 \%$ & $98.03 \%$ & $95.47 \%$ to $99.36 \%$ \\
\hline $\begin{array}{l}\text { Negative Predictive } \\
\text { Value }\end{array}$ & $91.57 \%$ & $88.47 \%$ to $94.06 \%$ & $94.37 \%$ & $91.43 \%$ to $96.53 \%$ \\
\hline
\end{tabular}

Fig.1 The various parameters that can be detected by using diagnostic testing methods along the time line of dengue infection

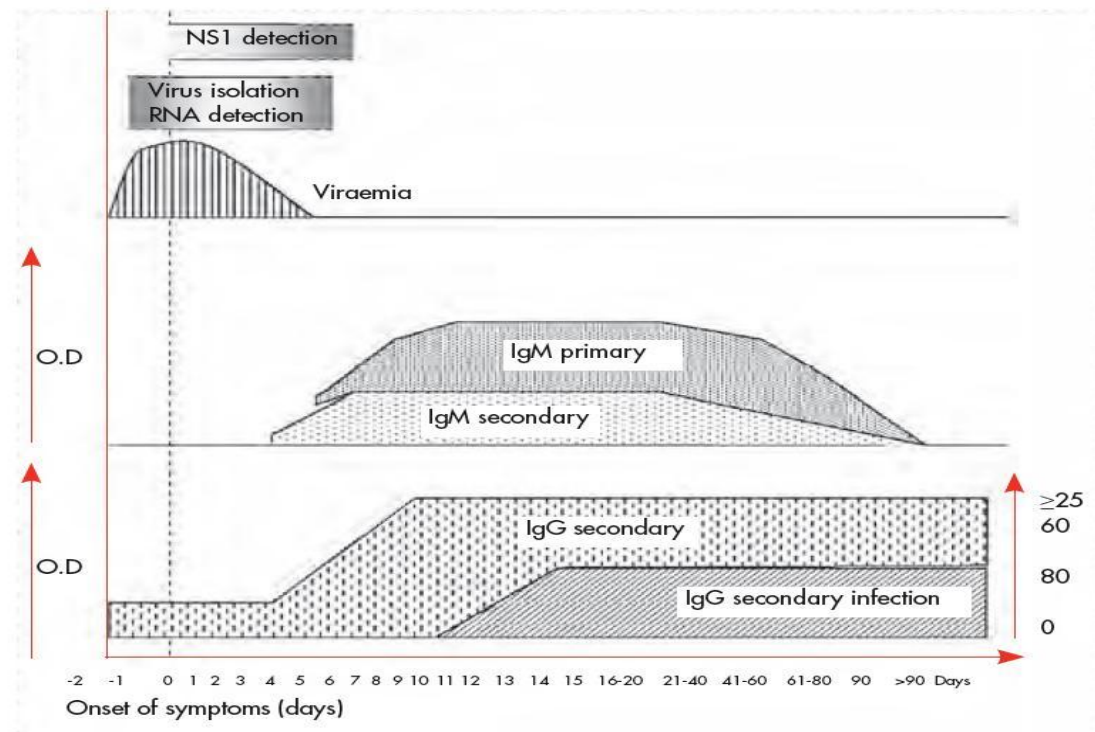

Fig.2 Age distribution of dengue cases confirmed by NS1 ELISA \& Ig M ELISA

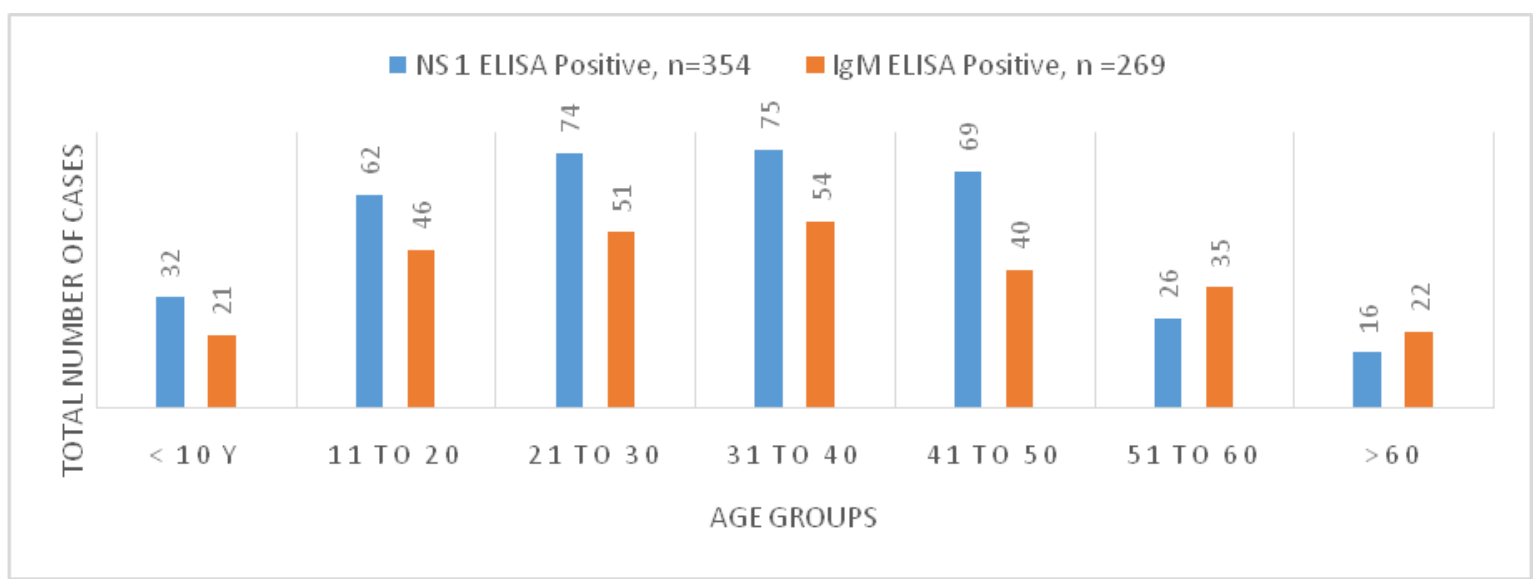


Fig.3 Gender distribution of the confirmed cases

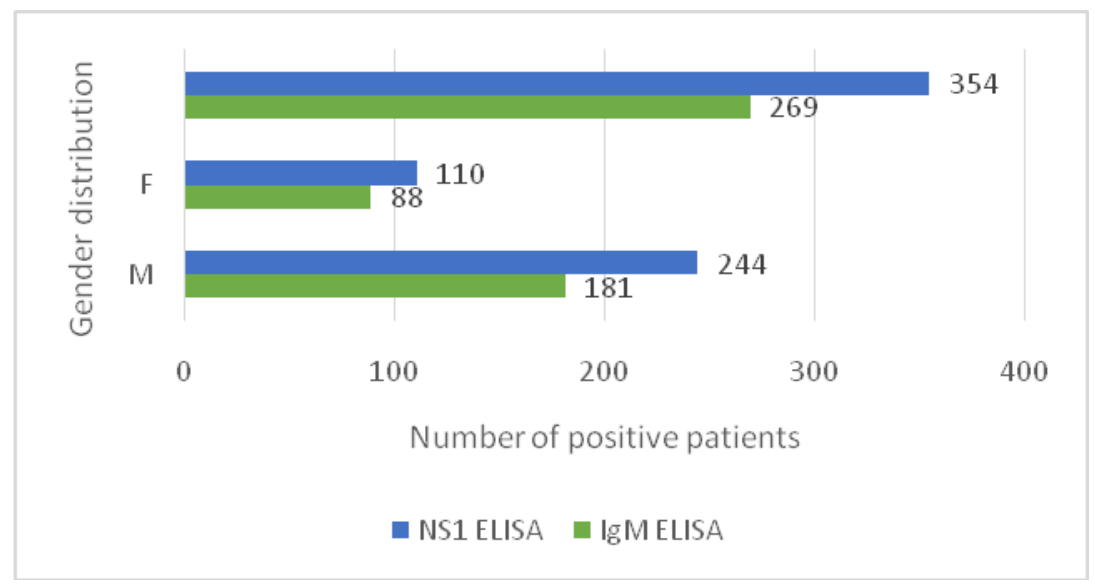

Fig.4 Day wise sensitivity and specificity of NS1 Ag rapid ICT vs NS1 antigen capture ELISA

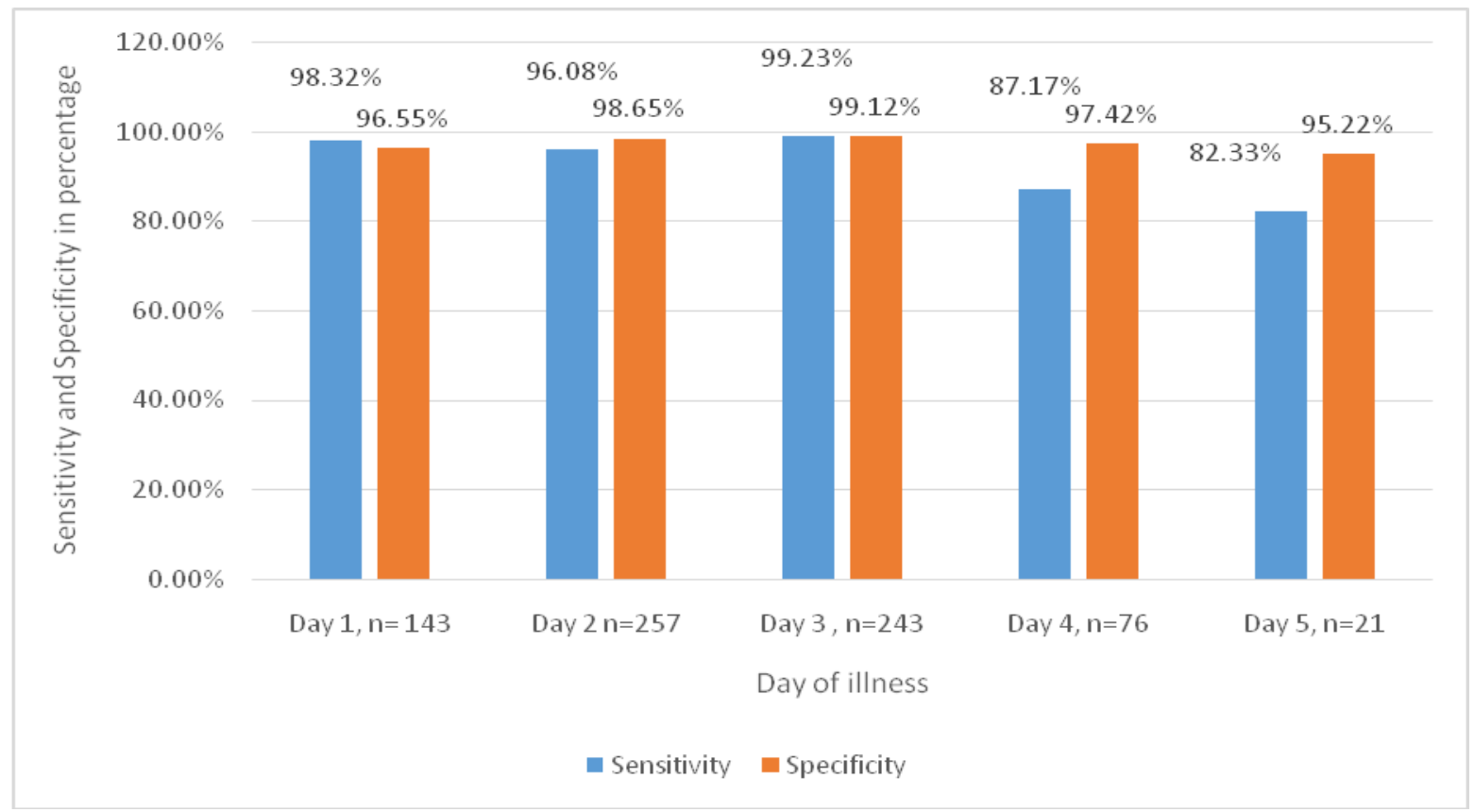

In comparison to ELISA, rapid ICT need very little technical expertise to perform and the turnaround time for the results is within minutes. Moreover, the main advantage of the rapid ICT is that a single sample can be run without waiting for the samples to be gathered and processed. Another advantage is that the combination test kits in which there is provision for performing both NS1 Antigen, IgM and IgGtests at one go are available(Mitra et al., 2016;Wang et al.,
2010). These kind of kits helps in shortening time for doing two different rapid tests and two different ELISA test, and can be used in situations where patients reporting on fourth or fifth day of fever and also in cases of secondary dengue infection with a different strain.

Our study showed that the rapid ICT kits that we used for testing NS1Ag and IgM performed at par with ELISA based tests. 
The sensitivity and specificity of various other kits in the market in a developing country like ours vary widely and this needs to be kept in mind while choosing and performing the dengue diagnostic tests. An initial validation of the rapid kits with the ELISA will definitely help. To conclude, in countries lacking infrastructure for the diagnostic labs especially in the rural and remote areas, the rapid dengue ICT tests can play a major role in diagnosis and in patient management of acute dengue infection. The rapid ICTs are very simple, easy to perform, and can be used as point of care tests. We suggest that the rapid ICT for NS1 Ag detection may be used in patients presenting with fever less than 5 days, rapid ICT for IgM antibody detection may be used in patients presenting with fever more than 5 days.

\section{Acknowledgements}

The authors thank all administrative authorities and laboratory staff and who helped us in carrying out this project.

\section{References}

Bhatt, Samir et al. 2013. "The Global Distribution and Burden of Dengue." Nature, $\quad 496(7446):$ 504-7. http://dx.doi.org/10.1038/nature12060.

Capeding, Maria Rosario, Z., et al. 2015. "Laboratory-Confirmed Dengue in Children in Three Regional Hospitals in the Philippines in 2009-2010." The Pediatric Infect. Dis. J., 34(11): 114551.

Chakravarti, A., R. Kumaria. 2005. "EcoEpidemiological Analysis of Dengue Infection during an Outbreak of Dengue Fever, India." Virol. J., 2. http://dx.doi.org/10.1186/ 1743-422X2-32.

Chaterji, Shera et al. 2011. "Evaluation of the NS1 Rapid Test and the WHO Dengue Classification Schemes for Use as Bedside Diagnosis of Acute Dengue Fever in Adults." The American J. Trop. Med. Hygiene, 84(2): 224-28.

Groen, Jan, et al. 2000. "Evaluation of Six Immunoassays for Detection of Dengue Virus-Specific Immunoglobulin $\mathrm{M}$ and $\mathrm{G}$ Antibodies." Clin. Diag. Lab. Immunol., 7(6): 867-71.

Gupta, Ekta, Lalit Dar, Geetanjali Kapoor, and Shobha Broor. 2006. "The Changing Epidemiology of Dengue in Delhi, India." Virol. J., 3(1): 1-5. http://dx.doi.org/10.1186/1743-422X3-92.

Mitra, Shubhanker, et al. 2016. "Comparative Evaluation of Validity and Cost-Benefit Analysis of Rapid Diagnostic Test (RDT) Kits in Diagnosis of Dengue Infection Using Composite Reference Criteria: A Cross-Sectional Study from South India." J. Vector Borne Dis., 53(1): 30-36.

National Guidelines for Clinical Management of Dengue Fever, Dec 2014.

http://www.nvbdcp.gov.in/iec.html.

NVBDCP (National Vector Borne Disease Control Programme). "Dengue Cases and Deaths in the Country since 2010." http://www.nvbdcp.gov.in/dencd.html.

Pal, Subhamoy, et al. 2014. "Evaluation of Dengue NS1 Antigen Rapid Tests and ELISA Kits Using Clinical Samples." PloS one, 9(11): e113411.

Sarkar, Arindam, Debjani Taraphdar, and Shyamalendu Chatterjee. 2012. "Molecular Typing of Dengue Virus Circulating in Kolkata, India in 2010." J. Trop. Med., 960329.

Shan, Xiaoyun et al. 2015. "Evaluation of 
the Diagnostic Accuracy of Nonstructural Protein 1 Ag-Based Tests for Dengue Virus in Asian Population: A Meta-Analysis." $B M C$ Infect. Dis., 15: 360.

Shih, Hsin-I et al., 2016. "Applications of a Rapid and Sensitive Dengue DUO Rapid Immunochromatographic Test Kit as a Diagnostic Strategy during a Dengue Type 2 Epidemic in an Urban City." PloS one, 11(7): e0158437.
Wang, Seok Mui, and Shamala Devi Sekaran. 2010. "Early Diagnosis of Dengue Infection Using a Commercial Dengue Duo Rapid Test Kit for the Detection of NS1, IGM, and IGG." The American J. Trop. Med. Hyg., 83(3): 690-95.

WHO. 2009. "Dengue: Guidelines for Diagnosis, Treatment, Prevention, and Control." Special Programme for Res. Training in Trop. Dis., 147.

\section{How to cite this article:}

Mahesh Reddy, R., Kavita Sahai, Ajay Mallik, S. Shoba and Anurag Khera. 2016. Comparative Analysis of Rapid Dengue Testing and ELISA for NS1 Antigen and IgM in Acute Dengue Infection. Int.J.Curr.Microbiol.App.Sci. 5(10): 931-937. doi: http://dx.doi.org/10.20546/ijcmas.2016.510.100 